\title{
Estado Actual de la Planificación de las Cuencas Hídricas en el Departamento de Sucre (Colombia)
}

\author{
Current State of Watersheds Planning in the Department of Sucre (Colombia)
}

\author{
Estado Atual do Planejamento das Bacias Hidrográficas no Departamento de Sucre (Colômbia)
}

\author{
Angelina María Hernández Arrázola. Ingeniera Civil \\ amha_13@hotmail.com \\ (iD) https://orcid.org/0000-0002-0297-8278 \\ Universidad de Sucre, Sincelejo, Colombia. \\ Keila Esther Landero Madera. Ingeniera Civil \\ keila.lanmad@gmail.com \\ (iD) https://orcid.org/0000-0001-8181-955X \\ Universidad de Sucre, Sincelejo, Colombia. \\ Juan Pablo Rodríguez Miranda. \\ jprodriguezm@udistrital.edu.co \\ (iD) https://orcid.org/0000-0002-3761-8221 \\ Universidad Distrital Francisco José de Caldas, Facultad del Medio \\ Ambiente y Recursos Naturales, Bogotá D.C., Colombia
}

Recibido: Mayo 8 de 2018

Aceptado: Noviembre 3 de 2018

Publicado: Diciembre de 2018

\section{RESUMEN}

El estado de las principales fuentes hídricas del Departamento de Sucre; el deterioro que se evidencia en la fauna, flora y poblaciones aledañas a estas; y la inexistencia de información sobre el manejo de territorios considerados como ecosistemas especiales, en este caso en particular, las áreas pertenecientes a cuencas hidrográficas, deja ver el estado de la planificación de las cuencas hídricas en el Departamento. Tal situación advierte la necesidad de una pronta ordenación, que permita mantener o restablecer el equilibrio entre el aprovechamiento de los servicios ecológicos de las cuencas hídricas y el estado natural de las mismas.

Palabras clave: Cuenca Hídrica; Precipitación; Planificación; Recursos naturales; Contaminación; Desarrollo Sostenible.

\section{ABSTRACT}

The state of the main water sources of the Department of Sucre; the deterioration that is evident in the fauna, flora and populations surrounding them; and the lack of information on the management of territories considered as special ecosystems, in this case in particular, the areas belonging to watersheds, shows the state of the planning of these in the Department. This situation warns of the need for a quick order, which allows maintaining or restoring the balance between the use of the ecological services of the water basins and the natural state of the same.

Keywords: Water basin; Precipitation; Planning; Natural resources; Pollution; Sustainable development.

\section{RESUMO}

O estado das principais fontes de água do Departamento de Sucre; a deterioração que é evidente na fauna, flora e populações que os rodeiam; e a falta de informação sobre a gestão de territórios considerados como ecossistemas especiais, neste caso em particular, as áreas pertencentes a bacias hidrográficas, mostra o estado do planejamento destes no Departamento. Esta situação alerta para a necessidade de um pedido rápido, que permita manter ou restabelecer o equilíbrio entre o uso dos serviços ecológicos das bacias hidrográficas e o estado natural dos mesmos.

Palavras-chave: Bacias Hidrográficas; Precipitação; Planejamento; Recursos naturais; Poluição; Desenvolvimento sustentável.

Como citar (APA)

Hernández Arrázola, A., Landero Madera, K., \& Rodríguez Miranda, J. (2018). Estado Actual de la Planificación de las Cuencas Hídricas en el Departamento de Sucre (Colombia). Procesos Urbanos 5:75-78. DOI: https://doi.org/10.21892/2422085X.412 


\section{Introducción}

Una Cuenca Hídrica corresponde a una zona en la cual, por sus características topográficas, se permite la confluencia de una o más fuentes hídricas. En "Fundamentals of Watershed Hydrology" (Edwards, 2015), se define una cuenca hidrográfica (Watersheds) como "un área en el terreno en la cual la precipitación drena en dirección a un mismo cuerpo de agua como resultado de su topografía". Una cuenca se alimenta principalmente a través de la precipitación y las escorrentías. En el Artículo 3, del Decreto 1640 de 2012, se define la cuenca hidrográfica como "el área de aguas superficiales o subterráneas que vierten a una red hidrográfica natural con uno o varios cauces naturales, de caudal continuo o intermitente, que confluyen en un curso mayor que, a su vez, puede desembocar en un río principal, en un depósito natural de aguas, en un pantano o directamente en el mar".

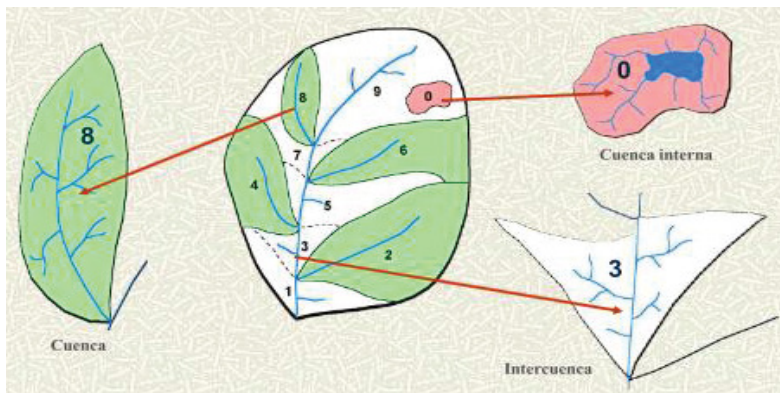

Figura 1. Cuenca Hidrográfica, Sistema de Codificación Estándar Internacional del Servicio Geológico de los EE.UU.

Fuente: Zonificación y Codificación de Cuencas Hidrográficas de Colombia (IDEAM, 2013).

Una cuenca u hoya hidrográfica se convierte, así, en un ecosistema donde el principal motor de funcionamiento es el agua, y donde se prestan servicios ecológicos de soporte, regulación, provisión y otros relacionados con el ámbito cultural, haciendo uso de los recursos naturales renovables y no renovables que hacen parte de esta.

\section{Identificación de Cuencas en el Departamento de Sucre}

En Colombia se han identificado como las zonas hidrográficas relevantes del territorio, cinco (05) macro-cuencas (Caribe, Magdalena-Cauca, Pacífico, Orinoco y Amazonas) las cuales cuentan con un complejo sistema hídrico. Cabe anotar que la topografía ha sido un factor importante en la identificación de las cuencas hídricas en el país, permitiendo la zonificación y caracterización de las mismas. El Departamento de Sucre, en el Caribe colombiano, se encuentra localizado en medio de dos de estas grandes hoyas hidrográficas: Caribe y Magdalena-Cauca.

Debido a la importancia de las cuencas hídricas en el contexto social, económico y ambiental de una región, y teniendo en cuenta el aumento en la demanda del recurso hídrico, el acceso limitado a este recurso por parte de muchas comunidades, las variaciones en la temperatura y el aumento incontrolado de los contaminantes, se concibe la necesidad de ordenar y planificar mejor el uso de este tipo de ecosistemas, para un adecuado aprovechamiento y conservación del mismo.

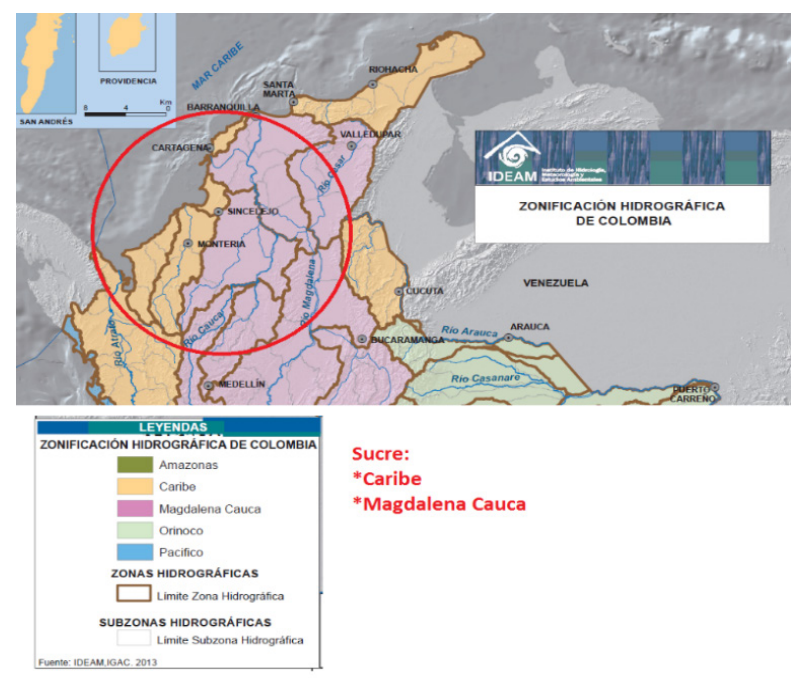

Figura 2. Departamento de Sucre en la Zonificación Hidrográfica de Colombia.

Fuente: Zonificación y codificación de cuencas hidrográficas de Colombia (IDEAM, 2013) 70249 .

\section{Normatividad para el Ordenamiento de las Cuencas en} Colombia

Considerando las leyes colombianas, como el Artículo 79 de la Constitución Política de Colombia, donde se establece que "Todas las personas tienen derecho a gozar de un ambiente sano. La ley garantizará la participación de la comunidad en las decisiones que puedan afectarlo. Es deber del Estado proteger la diversidad e integridad del ambiente, conservar las áreas de especial importancia ecológica y fomentar la educación para el logro de estos fines". Se denota que el criterio normativo para regular zonas especiales como cuencas hídricas, existe.

No es nuevo el tema de ordenamiento de las cuencas; remonta sus inicios en el Decreto Ley 2811 de 1974, en su Artículos 316 y 317 "316. Se entiende por ordenación de una cuenca la planeación del uso coordinado del suelo, de las aguas, de la flora y la fauna, y por manejo de la cuenca, la ejecución de obras y tratamientos. 317. Para la estructuración de un plan de ordenación y manejo, se deberá consultar a los usuarios de los recursos de la cuenca y a las entidades, públicas y privadas, que desarrollan actividades en la región". Luego, en la Ley 99 de 1993, donde se establecen las autoridades rectoras de dicha ordenación, en el año 2010, el Gobierno Nacional expide la Política Nacional para la Gestión Integral del Recurso Hídrico, hasta llegar al Decreto 1640 de 2012, "por medio del cual se reglamentan los instrumentos para la planificación, ordenación y manejo de las cuencas hidrográficas y acuíferos, y se dictan otras disposiciones". Aun cuando existe un paquete legal como herramienta para el ordenamiento funcional y coherente de las cuencas hidrográficas, en la actualidad el estado de estos instrumentos no está acorde con lo planteado normativamente, como lo es el caso de las cuencas hídricas identificadas en el territorio del Departamento de Sucre, el cual tiene influencia directa de los ríos Magdalena, Cauca y San Jorge en la Gran Cuenca de La Mojana Sucreña, además de hacer parte de la Cuenca del Caribe. 
Comúnmente, las áreas pertenecientes a una cuenca hidrográfica tienden a constituirse como centros de desarrollo para la región que la ocupa. Alejado de este contexto se encuentra el Departamento de Sucre, donde los municipios que lo conforman y hacen parte del territorio de una hoya hidrográfica son, precisamente, los más deprimidos, con poblaciones en condición de pobreza, con pocas fuentes de empleo y poca inversión del Estado. Por tanto, se desperdicia el potencial económico de estos ecosistemas, que al ser bien manejados $y$, por ende, organizados, permitirían avanzar en el desarrollo de este Departamento.

Solo hasta el año 2017, el Departamento de Sucre adopta el documento Formulación del Plan de Ordenación y Manejo de la Cuenca Hidrográfica La Mojana-Río Cauca "La Mojana agua y vida para todos", como el instrumento de manejo y ordenación para una de sus cuencas, la Gran Cuenca de La Mojana Sucreña, por medio del cual se espera que, una vez vigente el POMCA para esta, el panorama sea trasformado de manera positiva para las comunidades que hacen parte de este ecosistema.

\section{Desarrollo Sostenible Ideal versus Condiciones Actuales}

Enmarcados en el concepto de desarrollo sostenible, al planificar el manejo de una cuenca hídrica, se pretende el aprovechamiento de la misma sin agotar los recursos provistos por ella, previendo la permanencia de las condiciones naturales de servicios ecosistémicos para las generaciones futuras. En la actualidad, para el caso de La Mojana Sucreña, no existe un uso razonable de los recursos naturales, ya que se cuenta con presencia de mercurio contaminando las aguas $y$, a su vez, la flora, la fauna y poblaciones aledañas, produciendo enfermedades en las comunidades, como son los casos de malformación en menores. Pero el problema de la contaminación por mercurio no es lo único que afecta a La Mojana: los bosques están desapareciendo a causa de la deforestación progresiva por la expansión de la frontera agrícola; a esto se suma la caza incontrolada de animales silvestres, como el ponche y la hicotea, al igual que aves migratorias, como el barraquete.

En el caso de la Cuenca del Caribe en Sucre y su influencia sobre el Golfo de Morrosquillo, los daños ecológicos producidos por los derrames de petróleo corresponden a grandes emergencias ambientales. Estos sucesos han tenido efectos sobre la economía de los habitantes de esta zona costera, ocasionando disminución en la pesca y en las visitas turísticas, principales actividades económicas de la región. Adicional a esto, se presenta desforestación y relleno de humedales estuarios, múltiples puntos de vertimiento de aguas residuales sin tratamientos, entre otras problemáticas.

\section{Estado de la Planificación de las Cuencas en Sucre}

Las corporaciones que tienen jurisdicción sobre la Cuenca de La Mojana, mediante acto administrativo del 9 de enero de 2015, realizaron la declaratoria de estado de ordenamiento y formulación del plan de ordenación y manejo de esta cuenca, encontrándose en el detalle del proceso $\mathrm{N}^{\circ} \mathrm{CMA}-001-2015$. El contrato de consultoría se celebró con fecha de inicio de 20 de marzo de 2015, con un costo del proyecto de $\$ 2.016 .927 .600 .00$, con plazo de ejecución de 16 meses. Para el año 2017, se contó con el producto terminado y publicado (POMCA), acorde con lo establecido con las normas colombianas vigentes, y conforme con la situación de la cuenca, para su total aplicabilidad.

Caso contrario sucede con la Cuenca del Golfo de Morrosquillo, donde las autoridades competentes no han declarado esta cuenca en estado de ordenamiento, por falta de un consenso entre las diferentes corporaciones que tienen jurisdicción sobre ella. Sin embargo, el panorama planteado en el Golfo de Morrosquillo, incide en que los planes de ordenamiento de esta cuenca deben tener como eje enfrentar la problemática de deterioro ambiental de las zonas costeras, promoviendo su restauración, conservación y el manejo de los ecosistemas presentes.

\section{Conclusión}

Teniendo en cuenta todo lo anterior, se infiere que la normativa de planificación de una cuenca en el territorio colombiano sí existe y es aplicable. Sin embargo, parece desconocerse la importancia de este tipo de ecosistemas especiales en el desarrollo integral de una región, y no se toma en cuenta que estos son, en igual dimensión, tanto ricos en recursos como vulnerables al daño y al deterioro por las actividades antrópicas. Se ha menospreciado la necesidad de ordenación de las cuencas, tomándose hoy día como un documento requisito y no como una oportunidad de gestionar el buen uso del territorio y de los recursos disponibles en la región.

Actualmente, hace falta compromiso y responsabilidad por las autoridades competentes de la gestión, ordenación y la planificación de cuencas hídricas en el país, siendo este el caso del Departamento de Sucre, el cual, hasta el año 2017, contó con el Plan de Ordenamiento para la Cuenca de La Mojana-Rio Cauca, quedando en espera la gestión para el Ordenamiento de la Cuenca del Caribe, de la que hace parte el Departamento al tener influencia el Golfo de Morrosquillo. Los costos de esta planificación son elevados. Sin embargo, existe la necesidad de hacerlo para regular el buen uso de las cuencas. No está de más recomendar que se considere la formulación de políticas de uso y ocupación del territorio de cuencas hídricas, como un mecanismo para mantener y/o restablecer el equilibrio entre el aprovechamiento de los servicios ecosistémicos y su estructura natural.

Por otra parte, es preciso resaltar el valor que representa el POMCA de la Cuenca de La Mojana-Rio Cauca para el Departamento de Sucre, constituyéndose como la herramienta que, bajo una correcta aplicación y cumplimiento, permita regular y promover la formulación de proyectos en distintos sectores de desarrollo que se pretendan llevar a cabo dentro de las áreas de influencia de este ecosistema especial. 


\section{Referencias}

Constitución Política de Colombia (1991)

Sarmiento, C. H. S. (2016). Sustentabilidad y corporaciones autónomas regionales ambientales en boyacá - Colombia/Sustainability and environmental regional autonomous corporations in boyacá - Colombia. De https://search-proquest-com.ezproxy.cecar. edu.co:2443/docview/1768609054?accountid $=34487$

IDEAM (2013), zonificación y codificación de unidades hidrográficas e hidrogeológicas de Colombia, Bogotá, D. C., Colombia. Publicación aprobada por el Comité de Comunicaciones y Publicaciones del IDEAM, noviembre de 2013, Bogotá, D. C., Colombia.

Secretaría de la Convención de Ramsar, (2010). Manejo de cuencas hidrográficas: Integración de la conservación y del uso racional de los humedales en el manejo de las cuencas hidrográficas. Manuales Ramsar para el uso racional de los humedales, 4a edición, vol. 9. Secretaría de la Convención de Ramsar, Gland (Suiza).

Sotelo M.; Sotelo J. (2014). Planificación y gestión del agua en España en la actualidad Instituto Universitario de Ciencias Ambientales (IUCA/UCM) Departamento de Análisis Geográfico Regional y Geografía Física (UCM) Grupo de Investigación: "Desarrollo y Gestión Ambiental del Territorio". De file:///C:/Users/User/Downloads/47201-77937-2-PB.pdf

Salamanca C. (2015). Sustainability and environmental regional autonomous corporations in Boyacá - Colombia. De http://www.scielo. org.co/scielo.php?script=sci_arttext\&pid=S0120-30532016000100004

Edwards,P. Williard, K. Schoonover, Jon. (2015). "Guiding principles for management of forested, agricultural, and urban watersheds". De https://onlinelibrary.wiley.com/doi/full/10.1111/j.1936-704X.2015.03188.X

Edwards,P. Williard, K. Schoonover, Jon. (2015). "FUNDAMENTALS OF WATERSHED HYDROLOGY". De https://onlinelibrary.wiley.com/ doi/full/10.1111/j,1936-704X.2015.03185.X.

Edwards,P. (2015). "A primer on watershed management". De https://onlinelibrary.wiley.com/doi/full/10.1111/j,1936-704X.2015.03184.X

Floress K. Akamani K. Halvorsen K. Kozich4 A. Davenport M, (2015). "The role of social science in successfully implementing watershed management strategies". De https://www.fs.fed.us/nrs/pubs/jrnl/2015/nrs 2015 floress_001.pdf

Groninger J. (2015). "Building watershed management capacity in Nigeria: expanding the role of agriculture colleges". De https:// onlinelibrary.wiley.com/doi/pdf/10.1111/j,1936-704X.2016.03220.x

Cardona, L. (2001) "El enemigo oculto de la Mojana" El tiempo, http://www.eltiempo.com/archivo/documento/MAM-670227

El Tiempo (2004) La contaminación por la explotación oro tiene azotada la región de la Mojana, El tiempo http://www.eltiempo.com/ archivo/documento/MAM-1547788

U. T. Plan estratégico de las macrocuencas Magdalena-Cauca y Caribe (2013), "plan estratégico macro cuenca Caribe, Lineamientos y directrices de planificación estratégica"

CARSUCRE (2015), Contrato № 0072 "Consultoría para la elaboración (formulación) del plan de ordenación y manejo de la cuenca hidrográfica la Mojana - rio Cauca NSS (2502-02), en el marco del proyecto "Incorporación del componente de gestión del riesgo como determinante ambiental del ordenamiento territorial en los procesos de formulación y/o actualización de planes de ordenación y manejo de cuencas hidrográficas afectadas por el fenómeno de la Niña 2010-2011"

Decreto ley 2811. (1974), "Por el cual se dicta el Código Nacional de Recursos Naturales Renovables y de Protección al Medio Ambiente"

Ley 99. (1993), "Por la cual se crea el Ministerio del Medio Ambiente, se reordena el Sector Público encargado de la gestión y conservación del medio ambiente y los recursos naturales renovables, se organiza el Sistema Nacional Ambiental, SINA, y se dictan otras disposiciones"

Gobierno Nacional (2010), "Política Nacional para la Gestión Integral del Recurso Hídrico"

Decreto 1640. (2012), "Por medio del cual se reglamentan los instrumentos para la planificación, ordenación y manejo de las cuencas hidrográficas y Acuíferos, y se dictan otras disposiciones"

Corporación Autónoma Regional de Sucre, Corporación Autónoma Regional de Sur de Bolívar, Corporación Autónoma Regional del Canal del Dique resolución conjunta número 001 DE 2015, "por la cual se declara en Ordenación la Cuenca Hidrográfica La Mojana - Río Cauca NSS (Código 2502-02). 